\title{
Paediatric deep brain stimulation: ethical considerations in malignant Tourette syndrome
}

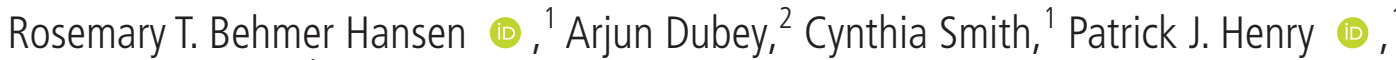 \\ Antonios Mammis ${ }^{1}$
}

${ }^{1}$ Department of Neurological Surgery, Rutgers New Jersey Medical School, Newark, New Jersey, USA

${ }^{2} \mathrm{~S}$ chool of Medicine, The University of Notre Dame Australia, Fremantle, Western Australia, Australia

\section{Correspondence to} Dr Antonios Mammis, Department of Neurological Surgery, Rutgers New Jersey Medical School, Newark, NJ 07103, USA;

mammisan@njms.rutgers.edu

Received 16 January 2020 Revised 29 February 2020 Accepted 27 March 2020 Published Online First 4 May 2020

Check for updates

(c) Author(s) (or their employer(s)) 2020. No commercial re-use. See rights and permissions. Published by BMJ.

To cite: Behmer Hansen RT, Dubey A, Smith C, et al.

$J$ Med Ethics

2020;46:668-673.

\section{ABSTRACT}

Gilles de la Tourette syndrome (TS) is a childhood neuropsychiatric disorder characterised by the presence of motor and vocal tics. Patients with malignant TS experience severe disease sequelae; risking morbidity and mortality due to tics, self-harm, psychiatric comorbidities and suicide. By definition, those cases termed 'malignant' are refractory to all conventional psychiatric and pharmacological regimens. In these instances, deep brain stimulation (DBS) may be efficacious. Current 2015 guidelines recommend a 6 -month period absent of suicidal ideation before DBS is offered to patients with TS. We therefore wondered whether it may be ethically justifiable to offer DBS to a minor with malignant TS. We begin with a discussion of non-maleficence and beneficence. New evidence suggests that suicide risk in young patients with TS has been underestimated. In turn, DBS may represent an invaluable opportunity for children with malignant TS to secure future safety, independence and fulfilment. Postponing treatment is associated with additional risks. Ultimately, we assert this unique risk-benefit calculus justifies offering DBS to paediatric patients with malignant TS. A multidisciplinary team of clinicians must determine whether DBS is in the best interest of their individual patients. We conclude with a suggestion for future TS-DBS guidelines regarding suicidal ideation. The importance of informed consent and assent is underscored.

\section{INTRODUCTION}

Gilles de la Tourette syndrome (TS) is a childhood neuropsychiatric disorder characterised by motor and vocal tics. ${ }^{1}$ While behavioural, psychological and pharmacological therapies offer satisfactory symptomatic relief for the majority of patients, approximately $5 \%$ of patients experience severe, refractory morbidity due to TS. ${ }^{2}$ These patients experience malignant TS. Deep brain stimulation (DBS) can reduce tic severity by approximately $50 \%$ in many of these patients. ${ }^{3}$ Nonetheless, there is a lack of detailed outcome data for paediatric patients with TS receiving DBS. DBS for paediatric patients is considered a surgical innovation. ${ }^{4}$

Current DBS guidelines for patients with TS were created by international experts, published in 2006 and updated in 2015. ${ }^{3}$ Indications include: (1) confirmed TS diagnosis; (2) proof that tics are the major source of impairment; and (3) condition is refractory to multiple pharmacological regimens. In addition, for at least 6 months prior to DBS, comorbid neuropsychiatric conditions should be stable, the patient must be actively compliant with psychological interventions, and there should be no substance abuse or suicidal ideation (SI). Patients should have social support and multidisciplinary team evaluation. If patients are under 18 years of age, guidelines recommend 'involving a local ethics committee or an institutional review board in the multidisciplinary evaluation'. ${ }^{35}$

We wondered whether a patient with severe, intractable TS may be offered DBS as a minor, or, instead, if DBS should be delayed until adulthood. Indeed, current reports suggest rates of suicide are even higher among young patients with TS than previously thought. ${ }^{6}$ After a review of TS and DBS, we outline key ethical considerations for treating patients with malignant TS. Specifically, we consider the bioethical principles of non-maleficence and beneficence. Ultimately, there are circumstances in which offering DBS to a minor with malignant TS is ethically justifiable. The role of informed consent is then discussed. Suggestions for future TS-DBS recommendations are shared. Because even medical professionals may perpetuate negative social stigmata towards patients with $\mathrm{TS}^{7}{ }^{7}$ we explicitly present up-to-date information regarding the condition at the beginning of this manuscript.

\section{TOURETTE SYNDROME}

TS is one of the most common childhood neuropsychiatric disorders, ${ }^{8}$ characterised by both motor and vocal tics for at least 1 year. ${ }^{9}$ Tics are sudden, rapid, recurrent and non-rhythmic movements or vocalisations. ${ }^{19}$ They are typically preceded by uncomfortable urges, and can be suppressed to varying degrees. ${ }^{710}$ In approximately $64 \%$ of TS cases, tics are painful. ${ }^{11}$ Tics can be simple or complex, infrequent or nearly continuous. ${ }^{12}$ Tics can cause severe emotional, social and psychological sequelae. ${ }^{11} 13$ They are exacerbated by anger, anxiety, excitement, stress, infections and elevated temperatures. ${ }^{1}$

Behavioural problems are often associated with TS. ${ }^{14}$ While $13 \%$ of cases lack associated comorbidities ('pure-TS'), the remaining approximately $87 \%$ of patients have comorbidities ('TS-plus'), ${ }^{11} 14$ including: attention deficit hyperactivity disorder (ADHD), obsessive-compulsive disorder (OCD), autism spectrum disorder, anxiety, depression, substance abuse, childhood conduct disorder, antisocial or disruptive behaviour, personality disorders as adults, mood disorders, schizotypal traits, sleep disorders and suicidal behaviours. ${ }^{11}$ Any of these comorbidities may impact quality of life and self-esteem. ${ }^{11}{ }^{14}$ Children with TS may experience bullying, isolation, loneliness and stigma. ${ }^{71}$ The peak severity of TS is in adolescence; threatening adjustment, maturation and psychosocial well-being. ${ }^{14}$ Many of these sequelae, particularly 
stigmatisation and discrimination, may result in decreased educational and employment opportunities persisting into adulthood. ${ }^{711}$ One study of patients with TS aged $16-54$ years found $29 \%$ experienced problems in family relationships and $27 \%$ had difficulties making friends. ${ }^{15}$ Patients with TS and/or chronic tic disorders (TS/CTD) experience a fourfold higher odds of dying by suicide than the general population. ${ }^{6}$

Approximately $1 \%$ of the US population has TS, ${ }^{14}$ with estimates as high as 3.8\% among those aged 5-18 years. ${ }^{13}$ Males are disproportionately affected. ${ }^{14}$ The mean age of onset is 7 years. ${ }^{14}$ TS is typically most severe during childhood and adolescence, with peak impairment around 10-12 years of age. ${ }^{13}$ Approximately one-third experience a significant decrease in tic severity in the third decade of life, ${ }^{112} 14$ with another one-third disappearing all together; termed the 'rule of three'. ${ }^{16}$ Unfortunately, the final third have fluctuating symptoms into adulthood. ${ }^{1}$ Even adults with prolonged periods of remission may experience a worsening of symptoms in adulthood. ${ }^{1}$ Prognostic factors predicting the course of TS for individual patients are lacking. ${ }^{512}$

\section{MALIGNANT TS AND DBS}

TS therapy is typically graded, including behavioural interventions and pharmacotherapy if necessary. ${ }^{1}$ While beyond the scope of this paper, readers are encouraged to review Deeb and Malaty's figure $2^{3}$ and Singer's figure $3-2^{1}$ for useful schematics of TS medication regimens. Recognition of comorbidities is critical in order to ensure that patient functioning reaches optimal levels. ${ }^{11}$

Approximately 5.1\% of patients are refractory to pharmacological and non-pharmacological treatments, termed to have 'malignant' TS. ${ }^{235}$ These patients have severe tics associated with self-injury, and are defined as having at least one hospitalisation or at least two emergency room visits due to TS symptoms or associated behavioural comorbidities. ${ }^{2} 316$ Patients with malignant TS tend to have self-injurious behaviour (SIB) $(64.7 \%)^{2}$ and complex phonic tics $(70.6 \%){ }^{2}$ An estimated $35.3 \%$ of patients with malignant TS have SI. ${ }^{2}$ Specific data regarding percentage of suicide attempts among this rare patient group are lacking. Persistent tics may result in disability ${ }^{13}{ }^{16}$ : documented cases include head-snapping tics resulting in cervical spinal cord injury ${ }^{10}$ or subdural haematoma, ${ }^{2}$ eye-poking tics causing blindness, ${ }^{10}$ bone fractures, ${ }^{5}$ retinal detachment ${ }^{5}$ and self-inflicted third-degree burns. ${ }^{2}$ Incidence rates for these lifethreatening sequelae, as well as morbidity and mortality rates, are unknown. ${ }^{2}$ By definition, therapies are not sufficient to alleviate the suffering among those patients with malignant TS. For these patients, DBS may be offered. ${ }^{35}$

DBS is a stereotactic surgery involving the implantation of an electrode into a target brain structure, which is connected to a programmable generator implanted into either the abdomen or chest. ${ }^{3}$ The implantable programmed generator (IPG) delivers an electrical signal into the brain in order to modulate electrical currents. ${ }^{3}$ DBS is approved by the Food and Drug Administration for treatment of Parkinson's disease, OCD, essential tremor and dystonia in adults; its use for paediatric patients is as a surgical innovation. ${ }^{34}$ Dystonia, in particular, is a movement disorder which can be treated with DBS in childhood, where earlier ages of treatment are associated with better outcomes. ${ }^{8}$ DBS was first used to treat TS in $1999 .^{3}$ It is believed that DBS is efficacious in TS because it targets structures in the cortico-basal gangliathalamocortical loop, which modulate movements. ${ }^{316}$ The most common targets for DBS in TS include the centromedian thalamus and the globus pallidus internus (GPi); however, many other targets have been used to treat TS. ${ }^{316}$ As in all DBS cases, patients with TS attend programming sessions for 3-6 months after surgery to optimise modulation. ${ }^{35}$

\section{NON-MALEFICENCE}

Non-maleficence refers to a clinician's obligation to avoid causing harm to their patients. DBS is associated with a few adverse effects, divided into surgical/hardware complications and stimulation complications. Use of DBS to treat paediatric patients is relatively rare, ${ }^{4} 17$ thereby limiting the quality of outcome data available. ${ }^{417}$ This challenges clinicians when performing risk-potential benefit analyses for their patients. ${ }^{4}$ In order to provide upmost transparency, we specify parameters for each statistic mentioned below.

The majority of adverse effects among patients receiving DBS of varying ages for TS are due to surgical and hardware complications, including infection rates of up to $18 \% .^{3}$ The postoperative infection rates for patients with TS may be higher than for others treated with DBS, as patients with TS may compulsively pick the hardware, causing skin erosions. ${ }^{3} 8$ Children receiving DBS for TS may experience higher rates of infection than adults, possibly due to the 'substantially smaller numbers of implanted patients' reported in existing studies, thereby decreasing the statistical power of those analyses. ${ }^{8}$ More accurate rates of paediatric infection after DBS will be generated with future, larger sample sizes. Additionally, infection rates may be higher among paediatric patients because leads and batteries used in all DBS procedures are sized for adults. ${ }^{818}$ The usual infection protocol for any patient with DBS requires removal of hardware and administration of antibiotics. ${ }^{8}$ Additionally, among patients with TS receiving DBS, there are rare reports of IPG malfunction or lead extension fractures, usually secondary to head-snapping tics. ${ }^{5}$

Overall, rates of adverse effects for patients of varying ages with TS receiving DBS are approximately $35.4 \%,{ }^{3}$ with variability explained by different DBS equipment, targets and stimulation parameters. In a meta-analysis by Coulombe et al of 58 paediatric patients aged $12-21$ years receiving DBS for TS, $27.6 \%$ of participants experienced side effects. These included: transient blurry vision (8.6\%), dysarthria (6.9\%), infection $(5.2 \%)$, anxiety $(5.2 \%)$, worsening of pre-existing tremor (3.4\%), dizziness (3.4\%), agitation (3.4\%) and a single patient each experienced haematoma, subcutaneous hydrops, hardware malfunction, lead tip cyst, lead fracture, tension headache, decreased memory, seizure-like episode, neck tightness, mild paraesthesia, light-headedness, parkinsonism, increased OCD, suicidal thoughts, seizure-like episode, disturbance of eye mobility and nausea. ${ }^{19}$ Among these patients, the GPi was the most common location for electrode placement. ${ }^{19}$ It is unclear whether any statistics gained from predominantly adult patient populations can be extrapolated to paediatric patient populations. $^{4}$

Cognitive decline has neither reliably nor consistently been associated with DBS in patients with TS. ${ }^{20}$ Long-term prospective data on paediatric patients who have received DBS for TS are lacking. Studies exist suggesting long-term utilisation of DBS for treatment of Parkinson's disease and dystonia may result in unwanted neural reorganisation. ${ }^{21} 22$ Their implications for clinical medicine are unclear. ${ }^{21} 22$ We agree with Davidson et al's perspective regarding DBS for paediatric patients: that consent discussions must always emphasise the lack of knowledge regarding long-term safety profile for DBS in rapidly growing 
brains. ${ }^{4}$ It is for this reason that DBS should be considered only for paediatric patients with severe, intractable TS.

And yet, the relatively reversible nature of DBS is important. The ability to adjust stimulation parameters enables many side effects to be mitigated for all patients receiving DBS. ${ }^{19} 23$ For example, among paediatric patients in Coulombe et al's analysis, all cases of blurry vision and both cases of dizziness were resolved by adjusting the stimulator voltage, while paraesthesia, light-headedness and nausea were all also related to stimulator parameters. ${ }^{19}$ Benefits, too, can be optimised. Limited evidence suggests children requiring DBS for TS may need more frequent programming than adults. ${ }^{5}$ Psychiatric comorbidities associated with TS and/or DBS may be treated with counselling and neuropsychiatric therapy. ${ }^{24}$ Possible harms may therefore not become permanent harms. In Coulombe et al's study, six paediatric patients $(10.3 \%)$ ultimately required lead removal, where of those two did so because their symptoms almost completely resolved after 4 and 5 years. ${ }^{19}$ We concur with published authors that DBS for patients with TS has minimal side effects and morbidity, including for children. ${ }^{4} 81924$

The risk of harm due to treating malignant TS with DBS must be compared with the risk of harm due to not treating malignant TS effectively; most seriously, self-harm. At the time of the recommendation update in $2015,{ }^{5}$ existing studies suggested that tic severity was associated with anxiety symptoms, which, in turn, were associated with SI. ${ }^{25}$ These studies argued that anxiety secondary to tic severity could be treated with traditional psychosocial methods, rendering DBS unnecessary to alleviate SI. ${ }^{5} 12$

Since the recommendation's publication, a new study revealed the relationship between TS and suicide was underestimated. A cohort study of the Swedish National Patient Register containing 7736 patients with TS/CTD from 1969 to 2013 demonstrated that compared with the general population, those with TS/CTD face a $439 \%$ increased higher odds of dying by suicide $(95 \% \mathrm{CI}$ 2.89 to 6.69 ) and a $386 \%$ increased odds of attempting suicide (95\% CI 3.50 to 4.26$).^{6}$ These risks persisted after adjusting for the presence of pre-existing psychiatric comorbidities. ${ }^{6}$ Tic presence beyond age 19 was a stronger predictor of death by suicide than having a previous suicide attempt as demonstrated by an 11.39 increased HR and a 5.65 increased HR, respectively (95\% CI 3.71 to 35.02 , and HR: 5.65; 95\% CI 2.21 to 14.42 , respectively). ${ }^{6}$ These findings offer a radically different prognosis for patients with TS: patients may face an increased risk of death by suicide due to tics alone, with increased risk when accounting for psychological comorbidities, social isolation and quality of life. The median age of suicide attempt was 20 and the median age of death was $31 .^{6}$

Fernández de la Cruz et al's findings emphasise a dire need for intervention in patients with TS before they reach adulthood. And yet because only $43.75 \%$ of those who died by suicide had a record of a previous attempt, ${ }^{6}$ we cannot assume those without a previous attempt are not at risk. Patients with TS/CTD were significantly more likely than controls to die via hanging, strangulation and suffocation ${ }^{6}$; methods that may cause substantial morbidity if not precipitating death. Reducing suicide risk requires managing psychiatric comorbidities and identifying factors contributing to SI. ${ }^{6}$ For many patients with TS, thorough medical and psychiatric evaluation and treatment is already sufficient to reduce SI. ${ }^{31119}$ Fernández de la Cruz et al provide us with evidence that, among those with TS who have SI refractory to medical and psychiatric treatment, tics alone may be the cause. Addressing and alleviating tics, therefore, may provide these patients with sufficient psychosocial relief to reduce their suicide risk. For most of these patients, traditional medical and psychiatric care will be sufficient to reduce their tics. For those whose tics are refractory to treatment, DBS may be considered as an effective means of reducing the psychosocial burdens caused by chronic tics. Out of 58 patients with TS aged 12-21 years who received DBS, $27 \%$ exhibited SIB. ${ }^{19}$

The consequences of not offering DBS to minors are likely higher than the above study estimates, as their sample included patients without malignant TS and with CTD in risk calculations. Patients with malignant TS are more likely to have multiple comorbidities than those without. ${ }^{2}$ They also have higher rates of SIB, behavioural comorbidities and legal issues. ${ }^{2}$ All of these factors are known contributors to SI, attempts and deaths. An estimated $35.3 \%$ of patients with malignant TS have SI. ${ }^{2}$

Non-maleficence involves consideration of both harms of offering DBS treatment, and harms of not offering DBS treatment. DBS carries risks of adverse events. Yet, DBS is generally less harmful than leaving patients with malignant TS without effective treatment. While many of the possible harms of DBS are reversible and can therefore be mitigated, many harms of malignant TS are not. Unfortunately, we lack prognostic factors to determine if TS will enter remission for any given patient and, if so, when and for what duration. Accurately determining harms currently experienced by each individual child with malignant TS is imperative. We concur with other authors that the risks of harming minors with malignant TS are higher when DBS is withheld than when DBS is administered. ${ }^{23}$ Current guidelines state for those with malignant TS, tics 'may carry greater risk of bodily harm, paralysis, or even death' than DBS. ${ }^{5}$ Better data must be collected on paediatric patients receiving DBS, ${ }^{2-4} 8$ particularly in order to optimise surgical targets, document and decrease adverse effects, and develop novel technology.

We believe addressing psychosocial concerns experienced by children with TS will help reduce these previously underrecognised suicide rates. Public health interventions to reduce TS myths and stigma may reduce the psychosocial burdens experienced by children with TS. ${ }^{6714}$ For those few patients whose tics and SI are both refractory to traditional medical and psychiatric treatment modalities, offering DBS may be ethically and medically appropriate.

\section{BENEFICENCE}

DBS must still provide measurable benefits to patients with malignant TS. This concept is grounded in the ethical obligation of beneficence. DBS is known to decrease tic symptomatology in patients with TS. These benefits are commonly measured using the Yale Global Tic Severity Scale (YGTSS), which is a semistructured clinical interview that measures tic severity and impairment. ${ }^{1}$ A decrease in YGTSS score by $25 \%$ is understood as a clinically meaningful improvement. ${ }^{3}$ The YGTSS measures, calculates and reports data in relative terms in order to compare functioning for each individual patient both before and after their DBS procedures. The YGTSS therefore offers special utility for clinicians, as they are able to directly assess the extent of benefits experienced by their patients.

Among patients with TS ranging from age 15 to 60 years, DBS has been documented to cause a median YGTSS reduction of $47.62 \%$ (38.8 points) with thalamic stimulation, and between $55 \%$ and 58\% (45.55-47 points) with GPi stimulation targets. ${ }^{326}$ Indeed, they found that patients of lower ages tended to experience greater YGTSS percentage reductions after DBS than those patients of older ages. ${ }^{26}$ And in Coulombe et al's meta-analysis of 58 patients aged 12-21 receiving DBS, 64\% experienced YGTSS 
score improvement of at least $50 \%$, no matter the target. ${ }^{19}$ Ninety-six per cent of children demonstrated at least some improvement in tic symptoms. ${ }^{19}$ Notably, the cohort's mean preoperative YGTSS score was 78.3+-17.5. ${ }^{19}$ DBS's impact on mood disorders in patients with TS is more variable. It may cause mood disorders, attenuate symptoms ${ }^{10}$ or even improve OCD and ADHD symptoms, ${ }^{25}$ all with substantial variability between individual patients. ${ }^{16}$ Benefits and patient satisfaction can be optimised through DBS programming. Reduction of tic severity may take at least 3 months after DBS implantation for patients with TS of various ages; taking approximately 13 months to decrease severity by $40 \% .^{3}$

What if the patient's TS would have gone into remission within a few years anyway? Given existing data, we believe it is irresponsible for clinicians to intentionally withhold treatment under the hope that malignant TS will spontaneously enter persistent remission. It is unclear whether the 'rule of three', which applies to TS patient prognosis in general, will equally apply to patients with malignant TS. While patients with malignant TS may be more likely than those with non-malignant TS to enter remission during adulthood, we find this implausible given the severity of malignant TS. ${ }^{2}$ Furthermore, even if patients with malignant TS were to experience complete remission in young adulthood, the mean age of TS onset for patients with malignant TS is 6.2 years. $^{2}$ Even if clinicians were certain that their patient's natural disease course would spontaneously remit during their 20 s, the profound psychosocial, academic, occupational and medical risks uniquely facing patients with malignant TS during the most important psychosocial developmental periods of their lives ${ }^{4}$ cause us to conclude that it is irresponsible to subject these children to years of suffering. Offering DBS to patients with intractable TS is ethically justified by the above risk-benefit calculus. Clinicians must determine whether DBS is indicated for a specific patient with malignant TS. ${ }^{4}{ }^{16}$ Characteristics to consider are outlined in detail in current TS-DBS recommendations. $^{512}$

\section{AUTONOMY AND DECISION-MAKING}

While we enumerated various harms and benefits, the decision to receive DBS is ultimately made by patients. It is therefore critical for clinicians to engage in a formal process of informed consent to elicit unique patient concerns, circumstances and values. Informed consent requires providing appropriate information about a proposed treatment, its risks and potential benefits, and the risks and potential benefits of any possible alternatives. ${ }^{27}$ The patient is able to ask questions and ultimately, voluntarily, decide. ${ }^{27}$ With DBS, informed consent should include a discussion of device insertion and possible removal in the instance of an adverse event. The discussion that follows refers to the US model of informed consent for clinical ethics.

There are standards for paediatric medical decision-making. Informed consent for minors requires both parental permission and patient assent. ${ }^{28} 29$ Assent allows a minor to agree to a medical intervention, even though they are not legally permitted to provide informed consent themselves. ${ }^{30}$ This approach provides minors with some autonomy. The goals of assent include: helping the child understand their condition; setting expectations; assessing their beliefs; and soliciting 'an expression of the patient's willingness to accept the proposed care' (all of which are possible in children as young as age 7). ${ }^{428}$

We now present scenarios to better illustrate ethical issues regarding DBS decision-making for a minor patient with malignant TS. The first scenario is if neither child nor parent wishes to receive DBS. Patients and their parents are able to give their informed refusal to a proposed treatment. In order to respect patient autonomy, physicians lack a right to force treatment on children in this instance. DBS must not be performed. The second circumstance is if both minor patient and parent desire DBS. Assuming parent or guardian gave their informed consent for DBS and the patients gave their assent, DBS may be administered. While informed consent is not a guarantee that a therapy will ultimately prove beneficial, patients retain the right to voluntarily accept certain risks. Importantly, DBS may only be performed if deemed a legitimate treatment option by a multidisciplinary team of clinicians. Patients do not have a positive right to demand treatment.

Circumstances of divergent parent-child wishes regarding DBS may arise. One example is if a parent wants DBS for their child, but the child does not provide their assent. Efforts must be made to improve communication between parent, child, caregivers and, if necessary, an ethics committee. A minor may only give their informed assent or refusal if they have some degree of decision-making capacity, as determined by their clinicians. To havecapacity, patients must be able to understand information, appreciate their current situation, demonstrate rational thinking and communicate a choice. ${ }^{31}$ We recommend using validated medical capacity assessment tools. In general, presence of a psychiatric condition-even if refractory to treatmentdoes not necessarily diminish a patient's capacity. ${ }^{23}$ If found that the minor has decision-making capacity, the patient should be able to give their informed refusal, grounded in the principle of bodily autonomy. If the minor lacks capacity, assent is generally necessary to treat children against their will, per the American Association of Pediatrics. ${ }^{28}$

Finally, a child may want DBS while the parent refuses. Again, maximal communication efforts should be made. If the disagreement persists, a few factors must be considered. First, if the patient is an emancipated minor, they are legally able to provide informed consent. ${ }^{32}$ Additionally, some states allow minors to receive medical treatment under the 'mature minor' exception. ${ }^{32}$ Regardless, however, the patient's decision-making capacity should be assessed.

The minor patient's desire to receive DBS can be ethically justified. Joel Fienberg's 'right to an open future' theory posits that children possess unique moral rights which forbid others from making decisions that limit their future opportunities. Malignant TS is associated with severe consequences including injuries, chronic pain, low quality of life, becoming housebound or institutionalised and unemployment. ${ }^{23}$ It is reasonable for a patient with malignant TS to value having future opportunities for work, housing, friendships, relationships and families. A dissenting parent may underestimate the severity of their child's condition, threatening the child's future derivation of safety, security, independence and fulfilment. Indeed, one study found that while $11 \%$ of parents reported their child with TS had SI symptoms, $61 \%$ of those children with TS actually had SI. ${ }^{25}$

Even if a minor has capacity to consent to DBS, practical barriers remain. First, adequate social support is currently necessary to receive DBS. DBS is a long-term commitment necessitating multiple device programming appointments. While the modifiable nature of DBS affords patients a unique ability to control their bodies, patients must be able to access surgery and appointments to realise these benefits. This is why social support is recommended. The second barrier is cost. Most minors cannot pay out of pocket for DBS and, instead are likely to be using their parents' health insurance. Minor patients wishing to receive DBS despite their parents' wishes may therefore need to wait until 
they reach the age of majority. Delaying therapy is associated with its own consequences, ${ }^{3-5} 16$ where meaningful and irreparable harms inflicted may disproportionately fall on patients.

Quantitative studies may uniquely demonstrate the decisionmaking processes that paediatric patients and their families experience when deciding whether or not to consent to DBS. Austin et al conducted such a study of DBS for children with dystonia. ${ }^{33}$ Their work is an excellent guide for researchers wishing to investigate the decision-making circumstances of patients with TS considering DBS.

\section{SUGGESTED RECOMMENDATION EDITS}

We agree with the 2015 recommendations, which state:

\begin{abstract}
The ideal DBS candidate will have a Diagnostic and Statistical Manual of Mental Disorders, Fifth Edition, diagnosis of TS and severe motor and vocal tics, which despite exhaustive medical and behavioural treatment trials result in significant impairment of selfesteem, social acceptance, family life, school or job functioning or physical well-being. ${ }^{5}$
\end{abstract}

We now suggest a single edit. The recommended 6-month period of no SI before being considered for DBS appears to be well intentioned, justified by concerns of poor follow-up, ${ }^{5}$ compromised outcome assessment ${ }^{512}$ and poor adaptation to adverse events. Nonetheless, we believe it may be harmful in light of new evidence. Approximately one-third of patients with malignant TS have documented SI. Because TS is a childhoodonset condition, this recommendation may be placing paediatric patients with TS at unique risks of harm. By decreasing tic severity, DBS addresses the root cause of SI for some patients, as described in the non-maleficence discussion. Existing guidelines delay potential benefits beyond those delays intrinsic to DBS therapy by excluding many of these patients from receiving potentially life-saving treatment for at least 6 months. We suggest this recommendation is revisited. We believe all other current recommendations remain appropriate, particularly for paediatric patients with malignant TS. While beyond the scope of this manuscript, we affirm the need for more research and public health interventions to reduce suicidality among patients with chronic tics. Factors such as cost, patient accessibility and outcomes should be considered.

\section{CONCLUSIONS}

The stakes are high. Years of childhood TS are associated with significant medical and psychosocial sequelae that threaten both present and future functioning. ${ }^{3}$ For the small subset of patients with malignant TS, DBS represents an invaluable opportunity to decrease profound morbidity and mortality.

Current recommendations have laid a strong foundation for DBS decision-making. In this article, we sought to build on these by explicitly articulating the ethical dimensions of decisionmaking for patients with malignant TS, their families and their multidisciplinary team of clinicians. It is our privilege to highlight exceptional interdisciplinary research, and our hope that it will be considered in the creation of future recommendations.

With critical and conscientious eyes, we can continue to improve care for patients with TS through the judicious incorporation of new knowledge and technology. It follows that future TS-DBS recommendations may be improved by re-evaluating their current position regarding SI, as it may place paediatric patients at unique risk. Future research enumerating risk factors for poor TS prognosis is needed, particularly for paediatric populations.

Contributors $A M$ and RTBH had the idea for the article. RTBH, AD, CS and PJH performed the literature search. RTBH, AD, CS and PJH wrote the article. AM and RTBH are the guarantors.

Funding The authors have not declared a specific grant for this research from any funding agency in the public, commercial or not-for-profit sectors.

Competing interests None declared.

Patient consent for publication Not required.

Provenance and peer review Not commissioned; externally peer reviewed.

\section{ORCID iDs}

Rosemary T. Behmer Hansen http://orcid.org/0000-0002-6335-7627

Patrick J. Henry http://orcid.org/0000-0002-5690-7539

\section{REFERENCES}

1 Singer HS. Tics and Tourette syndrome. Continuum 2019;25(4):936-58.

2 Cheung M-YC, Shahed J, Jankovic J. Malignant Tourette syndrome. Mov Disord 2007;22(12):1743-50.

3 Deeb W, Malaty I. Deep brain stimulation for Tourette syndrome: potential role in the pediatric population. J Child Neurol 2020;35(2):155-65.

4 Davidson B, Elkaim LM, Lipsman N, et al. Editorial. An ethical framework for deep brain stimulation in children. Neurosurg Focus 2018;45(3):E11.

5 Schrock LE, Mink JW, Woods DW, et al. Tourette syndrome deep brain stimulation: a review and updated recommendations. Mov Disord 2015;30(4):448-71.

6 Fernández de la Cruz L, Rydell M, Runeson B, et al. Suicide in Tourette's and chronic tic disorders. Biol Psychiatry 2017;82(2):111-8.

$7 \mathrm{Cox} \mathrm{JH}$, Nahar A, Termine C, et al. Social stigma and self-perception in adolescents with Tourette syndrome. Adolesc Health Med Ther 2019;10:75-82.

8 Lipsman N, Ellis M, Lozano AM. Current and future indications for deep brain stimulation in pediatric populations. Neurosurg Focus 2010;29(2):E2.

9 Eaton CK, Jones AM, Gutierrez-Colina AM, et al. The influence of environmental consequences and internalizing symptoms on children's tic severity. Child Psychiatry Hum Dev 2017:48(2):327-34.

10 Leckman JF. Deep brain stimulation for Tourette syndrome: lessons learned and future directions. Biol Psychiatry 2016;79(5):343-4.

11 Eapen V, Cavanna AE, Robertson MM. Comorbidities, social impact, and quality of life in Tourette syndrome. Front Psychiatry 2016;7(97).

12 Mink JW, Walkup J, Frey KA, et al. Patient selection and assessment recommendations for deep brain stimulation in Tourette syndrome. Mov Disord 2006;21(11):1831-8.

13 Edwards KR, Mendlowitz S, Jackson E, et al. A qualitative exploration of the experiences of children and adolescents with Tourette syndrome. J Can Acad Child Adolesc Psychiatry 2017;26(1):39-44

14 Silvestri PR, Chiarotti F, Baglioni V, et al. A preliminary examination of self-concept in older adolescents and young adults with Gilles de la Tourette syndrome. Eur J Paediatr Neurol 2017;21(3):468-74.

15 Muller-Vahl KR. Deep brain stimulation in Tourette syndrome: the known and the unknown. J Neurol Neurosurg Psychiatry 2019;90(10):1076-7.

16 Xu W, Zhang C, Deeb W, et al. Deep brain stimulation for Tourette's syndrome. Trans/ Neurodegener 2020;9(1):1-19.

17 Hale AT, Monsour MA, Rolston JD, et al. Deep brain stimulation in pediatric dystonia: a systematic review. Neurosurg Rev 2018. doi:10.1007/s10143-018-1047-9. [Epub ahead of print: 05 Nov 2018].

18 Marks WA, Honeycutt J, Acosta F, et al. Deep brain stimulation for pediatric movement disorders. Semin Pediatr Neurol 2009;16(2):90-8.

19 Coulombe M-A, Elkaim LM, Alotaibi NM, et al. Deep brain stimulation for Gilles de la Tourette syndrome in children and youth: a meta-analysis with individual participant data. J Neurosurg 2019;23(2):236-46.

20 Cernera S, Okun MS, Gunduz A. A review of cognitive outcomes across movement disorder patients undergoing deep brain stimulation. Front Neurol 2019;10(10):419.

21 Ruge D, Cif L, Limousin $\mathrm{P}$, et al. Shaping reversibility? long-term deep brain stimulation in dystonia: the relationship between effects on electrophysiology and clinical symptoms. Brain 2011;134(Pt 7):2106-15.

22 Ruge $D$, Cif L, Limousin P, et al. Longterm deep brain stimulation withdrawal: clinical stability despite electrophysiological instability. J Neuro/ Sci 2014;342(1-2):197-9.

23 Grant RA, Halpern CH, Baltuch GH, et al. Ethical considerations in deep brain stimulation for psychiatric illness. J Clin Neurosci 2014;21(1):1-5.

24 Kakusa B, Saluja S, Tate WJ, et al. Robust clinical benefit of multi-target deep brain stimulation for treatment of Gilles de la Tourette syndrome and its comorbidities. Brain Stimul 2019;12(3):816-8.

25 Johnco C, McGuire JF, McBride NM, et al. Suicidal ideation in youth with tic disorders. J Affect Disord 2016:200:204-11.

26 Baldermann JC, Schüller T, Huys D, et al. Deep brain stimulation for TouretteSyndrome: a systematic review and meta-analysis. Brain Stimul 2016;9(2):296-304.

27 Berg JW, Appelbaum PS, Lidz CW, et al. Informed consent: legal theory and clinical practice. 2nd ed. Fair Lawn: Oxford University Press, 2001. 
28 Katz AL, Webb SA, on B C. Informed consent in decision-making in pediatric practice. Pediatrics 2016;138(2):e20161485.

29 The American Academy of Pediatrics. Informed consent, parental permission, and assent in pediatric practice. Pediatr 1995;95(2):314 LP-7 http://pediatrics. aappublications.org/content/95/2/314.abstract

30 De Lourdes Levy M, Larcher V, Kurz R, et al. Informed consent/assent in children. statement of the ethics Working group of the Confederation of European specialists in paediatrics (CESP). Eur J Pediatr 2003;162(9):629-33.
31 Appelbaum PS, Grisso T. Assessing patients' capacities to consent to treatment. N Engl J Med 1989:320(11).

32 Coleman DL, Rosoff PM. The legal authority of mature minors to consent to general medical treatment. Pediatrics 2013;131(4):786-93.

33 Austin A, Lin J-P, Selway R, et al. What parents think and feel about deep brain stimulation in paediatric secondary dystonia including cerebral palsy: a qualitative study of parental decision-making. Eur J Paediatr Neurol 2017;21(1):185-92. 